\title{
Desarrollo de la escritura mediante la implementación de estrategias didácticas en inglés, grado noveno
}

\author{
Clara Eidelsa Villamizar \\ clara.villamizar3@unipamplona.edu.com \\ Universidad de Pamplona \\ Pamplona-Colombia \\ Carlos Alberto Jaimes \\ cjaimes1@gmail.com \\ Universidad De Pamplona
}

\section{RESUMEN}

Esta investigación-acción tuvo como objetivo: Desarrollar habilidades de escritura en inglés, mediante la implementación de estrategias didácticas, en estudiantes de noveno grado del Colegio Puerto Santander. Los objetivos específicos fueron: Identificar el interés que poseen los estudiantes de noveno grado para la escritura en inglés. Potenciar la producción textual de los estudiantes mediante estrategias de escritura basada en sus experiencias cotidianas. Por consiguiente, la metodología empleada es una investigación cualitativa, la recolección de datos y por medio de técnicas como la entrevista, el cuestionario, diario de campo, luego, por medio del análisis de datos, determinar el nivel de desempeño en producción escrita de los estudiantes e identificar características claves para los talleres y su evaluación. Para tal fin, se implementaron estrategias a través de seis secuencias pedagógicas a una muestra de cinco estudiantes. En conclusión, las estrategias didácticas para mejorar la escritura en los estudiantes, resultaron exitosas como: la reescritura, que involucra la toma de notas en clase, el uso del diccionario bilingüe, la estrategia de memorización, como los ejercicios en grupo, exposiciones orales, ete, la retroalimentación, el cual el estudiante puede corregirse y corregir a sus compañeros. Al igual que con la ayuda de las herramientas tecnológicas el internet

Palabras clave: Estudiantes; escritura; estrategias; habilidades; productividad 


\title{
Writing development through the implementation of teaching strategies in english, ninth grade
}

\begin{abstract}
The objective of this action research was: To develop writing skills in English, through the implementation of didactic strategies, in ninth grade students of Colegio Puerto Santander. The specific objectives were: Identify the interest that ninth grade students have for writing in English. Enhance students' textual production through writing strategies based on their everyday experiences. Therefore, the methodology used is a qualitative research, data collection and through techniques such as interviews, questionnaires, field journals, then, through data analysis, determine the level of performance in written production of the students and identify key characteristics for the workshops and their evaluation. To this end, strategies were implemented through six pedagogical sequences to a sample of five students. In conclusion, the didactic strategies to improve the students' writing were successful, such as: rewriting, which involves taking notes in class, the use of the bilingual dictionary, the memorization strategy, such as group exercises, oral presentations, ete, feedback, which the student can correct himself and his classmates. As with the help of technological tools the internet
\end{abstract}

Keywords: Strategy; Students; Writing; Skills; Productivity

Artículo recibido: 27 marzo 2021 Aceptado para publicación: 30 abril 2021 Correspondencia: clara.villamizar3@unipamplona.edu.com

Conflictos de Interés: Ninguna que declarar 


\section{INTRODUCCIÓN}

Con la realización del presente estudio se busca desarrollar la escritura de manera competitiva, por medio de la implementación de estrategias didácticas para el aprendizaje efectivo de la asignatura del inglés en estudiantes de noveno grado del Colegio Puerto Santander. Así mismo, el desarrollo de estrategias didácticas en la escritura del inglés es esencial para todo tipo de comunicación: lectura, habla, escucha y obviamente escritura. Para Santana \& et al. (2016) señalan que el "aprendizaje del idioma inglés cobra cada vez mayor auge en el mundo, sin embargo, la brecha entre la calidad del aprendizaje de una segunda lengua entre los estudiantes es cada vez mayor" (p. 80). Para esta investigación se realizó un análisis de varios estudios relacionados con el tema de la escritura, así como con la apropiación de las diferentes estrategias metodológicas. De igual importancia, uno de estos estudios es el de Veloz, S. (2015) El Trabajo Autónomo en los Estudiantes de Inglés como parte de sus procesos de aprendizaje en la habilidad escrita, en el Semestre "A" Año 2014 de los estudiantes en el centro de idiomas extranjeros de la Universidad católica de Santiago de Guayaquil. Maestría en Educación superior. Guayaquil, Ecuador. Así pues, esta investigación tiene el propósito de mejorar el trabajo autónomo para los estudiantes que deseen aprender por sí mismos, interesados en mejorar la autonomía de su aprendizaje en su habilidad escrita del idioma inglés.

En primer lugar, aprender una lengua extranjera es una necesidad que crece día a día, lo que hace que la sociedad actual y las nuevas tecnología exijan a los estudiantes manejar una segunda lengua, en este caso el inglés (Crystal, 1997) que "les permitan tener acceso a una cantidad enorme de información de diferentes áreas del conocimiento, que viene dada en otros idiomas". De esta forma, el estudio se centró principalmente en generar estrategias didácticas, para motivar en los jóvenes de Noveno grado la habilidad de la escritura de pequeños textos de inglés, durante el año escolar.

Dentro de las habilidades que el estudiante debe ejecutar, la escritura, objeto de este proyecto de investigación, es una de las formas vigentes de la comunicación, sin embargo, no es para algunos la forma más fácil de interactuar, pues requiere de elementos formales para que la comunicación sea exitosa. En Colombia existen estrategias de bilingüismo que promueven a las instituciones educativas a ampliar las capacidades expresivas en inglés con el objeto que al terminar los estudios de básica primaria y media vocacional los estudiantes sean más competitivos frente a una 
colectividad que cada vez más está ligada al inglés en la parte política, social, cultural, y económica.

De esta manera, es importante que todos los docentes motiven al estudiante a la lectura, como ayuda de comprensión y producción escrita, porque el estudiante que es capaz de escuchar y pronunciar bien, puede leer y logra incrementar el vocabulario, escribir correctamente; por lo tanto, debe producir documentos escritos. Además, las fortalezas encontradas en relación con la motivación de los estudiantes, la satisfacción por la asignatura de inglés y su interés por las nuevas técnicas y recursos son factores que potencializados contribuirán al desarrollo de la autonomía. Por consiguiente, los docentes deben de apreciar la capacidad de los estudiantes para aprender, Es decir usar los conocimientos y aprendizaje que poseen los estudiantes para mejorar su propio aprendizaje en la asignatura de inglés en la habilidad escrita. En otras palabras, los docentes deben fortalecer a través de sus técnicas y recursos utilizados en el curso de inglés, que los estudiantes a través de su trabajo autónomo conserven el dominio de su propio aprendizaje y puedan realizar producción de sus conocimientos. Por otro lado, deben aplicar instrumentos validados para evaluar la preparación del estudiante para el aprendizaje autónomo, lo que demanda que el profesor debe ser constante, no solo en términos de conocimientos sino de la práctica pedagógica para ayudar al estudiante a un mejor conocimiento y de comprometerse a alcanzar niveles más profundos $\mathrm{y}$ significativos en el proceso de aprendizaje. igual forma, se encuentra la investigación de Guano, D. \& et al. (2017) el aprendizaje significativo como estrategia de estimulación de la escritura del Idioma Inglés en educación general básica, donde mostró el análisis del uso de estrategias de aprendizaje significativo para la estimulación de la escritura del idioma inglés. Así, se evidencia la falta de la estrategia de aprendizaje y que afecta el desarrollo de la escritura y que causa bajo rendimiento y deficiente nivel de conocimientos en los estudiantes de sextos y séptimos años de educación general básica.

Encontramos a Rincón, A. (2013) donde expresa en el trabajo de tesis titulado fortalecimiento de la escritura en inglés en estudiantes de educación media de una institución pública, que "El planteamiento del problema gira alrededor del proceso de enseñanza y aprendizaje de la lengua inglesa a través del desarrollo de la competencia comunicativa, fortaleciendo el proceso de escritura el cual no se da tan fácilmente, ya 
que esta habilidad requiere de algún tipo de aprendizaje y donde inicialmente se enfoca en los procesos cognitivos de la escritura para realizar composiciones en inglés" (p.15). Es decir, que la metodología es de tipo descriptivo desde el paradigma cualitativo. De ahí que la muestra seleccionada y grupo piloto se conformó con seis estudiantes de grado $9^{\circ}$. Al igual, las técnicas seleccionadas para la recolección de datos fueron la encuesta, la entrevista, la observación durante seis sesiones y los documentos realizados por los participantes durante el acto de composición en inglés. Enseguida, se efectúa luego un trabajo de análisis en espiral y de manera dialéctica por medio de comparaciones del antes y el después del uso de los procesos cognitivos implicados en la escritura, en las composiciones hechas por los participantes, CER Urbano Ruiz del municipio de Amagá, inicialmente se hizo una caracterización de las propuestas didácticas empleadas en la enseñanza del inglés. Por ello, se tiene en cuenta que escribir es una habilidad que se incrementa a medida que el estudiante se arriesga a hacerlo, Igualmente, no es posible desear aprender a escribir si no se hace el ejercicio de escribir. Por lo tanto, el propósito de la investigación, es definir y poner en práctica las estrategias adecuadas que conlleven al estudiante a mejorar su escritura en inglés y que su práctica lo hagan ser funcional en el mundo globalizado actual.

\section{ESTRATEGIAS METODOLÓGICAS O MATERIALES Y MÉTODOS}

La metodología del estudio se inclina por el paradigma cualitativo, el cual describe una realidad social, descriptiva e interpretativa, según Sampieri (2006), "La investigación cualitativa se centra en comprender y profundizar los fenómenos, explorándolos desde la perspectiva de los participantes en un ambiente natural y en relación con el contexto" (p.23). De hecho, la información obtenida describe los componentes básicos del proceso estudiado, la cual se analiza con el fin de establecer las características requeridas para elaborar conclusiones. Por ello la investigación se considera también como investigación acción- participante.

La recolección de datos se realiza por medio de técnicas como la entrevista, el cuestionario, diario de campo y después analizar, por medio de la técnica del análisis contenido, el nivel de desempeño en producción escrita de los estudiantes e identificar algunas características comunes que resulten claves para el diseño de talleres y su evaluación. Para tal fin, se implementaron una serie de estrategias a través de seis secuencias pedagógicas en el aula a una muestra de cinco estudiantes. 
En el estudio, se utilizaron instrumentos no estandarizados, pero de contenido muy significativo para el estudio, que no tiene carácter comparativo con otra población. Se aplicó un cuestionario que consta de doce preguntas de doble opción: Falso (F) y Verdadero (V). Se realizó una entrevista personal, que se centra en diez aspectos pertinentes al fenómeno estudiado con opciones abiertas para la respuesta. Igualmente se utilizó un diario de campo, que recogió los acontecimientos más relevantes y pertinentes que ocurrieron durante los talleres de intervención realizados durante la segunda fase del estudio.

En el diario de campo se registró para cada uno de los talleres de composición escrita con detalles relevantes para trabajar otro de los objetivos de la investigación. Una vez aplicado el cuestionario se revisó para obtener las respectivas frecuencias de respuestas y se construyó una tabla, la cual se analiza punto por punto. Seguidamente, las entrevistas se digitaron y para su análisis se establecieron categorías de acuerdo a la información recibida, teniendo en cuenta que a cada pregunta se le hizo su análisis respectivo, resaltando uno de los objetivos de la investigación.

El diario de campo según Nichol (2006), este instrumento "proporciona acceso al mundo subjetivo de las personas, desarrolla imagines realistas y proporciona descripciones sensitivas de la vida diaria de un individuo, y crea un archivo completo de acciones” (p.51). Según lo expresa Galeano (2018), la organización de la información depende de aspectos como "el enfoque utilizado, la realidad de la investigación, el objeto de estudio, el propósito de la misma, entre otras. También se utilizó un cuestionario" (p. 7), que según Galán Amador (2009)

"El cuestionario es un conjunto de preguntas diseñadas para generar los datos necesarios para alcanzar los objetivos propuestos del proyecto de investigación. Entonces, el cuestionario permite estandarizar e integrar el proceso de recopilación de datos; sin embargo, un diseño mal construido e inadecuado conlleva a recoger información incompleta, datos no precisos de esta manera genera información nada confiable. Por esta razón, el cuestionario es en definitiva un conjunto de preguntas respecto a una o más variables que se van a medir" (p.69).

En el estudio se realizó una entrevista semiestructurada, que para Denzin \& Lincoln (2005, tomado de Vargas, 2012) la entrevista "es el arte de realizar preguntas y escuchar respuestas" (p.643). Esta definición, demasiado genérica y poco operativa, incluye cualquier encuentro entre dos personas, un investigador y su entrevistado, en el que el 
investigador hace preguntas que pueden ir desde las encuestas de opinión o los cuestionarios, es decir, instrumentos altamente estructurados, y las entrevistas abiertas donde es posible, incluso, que el investigador sea preguntado e interpelado por el informante. (Sabino, 1992) comenta que la entrevista, desde el punto de vista del método es una forma específica de interacción social que tiene por objeto recolectar datos para una investigación. Seguidamente, el investigador formula preguntas a las personas capaces de aportarle datos de interés, estableciendo un diálogo peculiar, asimétrico, donde una de las partes busca recoger informaciones y la otra es la fuente de esas informaciones.

Cabe destacar que, la ventaja esencial de la entrevista radica en que son los mismos actores sociales quienes proporcionan los datos relativos a sus conductas, opiniones, deseos, actitudes y expectativas, cosa que por su misma naturaleza es casi imposible de observar desde fuera. Es decir, que nadie mejor que la misma persona involucrada para hablarnos acerca de todo aquello que piensa y siente, de lo que ha experimentado o proyecta hacer (Cassany, 2002).

De hecho, el estudio se llevó a cabo con los estudiantes de Grado 9-01, en dos fases, la primera consistió en la fase descriptiva diagnóstica. En esta etapa se observó a los estudiantes en su contexto, se tuvo en cuenta su nivel de aprendizaje de la lengua inglesa, su entorno estudiantil y familiar, su actitud y disposición hacia la clase de inglés. La segunda fase, correspondió a la intervención propiamente dicha, que estuvo conformada por seis sesiones debidamente planeadas y en la que los actores (participantes clave) jugaron un papel protagónico, tanto en su planeación como en su ejecución y valoración. Además, en esta fase se permitió valorar la producción textual de los estudiantes mediante estrategias de escritura creativa basadas en sus experiencias cotidianas.

Con base en la información recolectada y con apreciaciones como docente de inglés de los estudiantes de noveno grado 01, se desarrollaron una serie de talleres pedagógicos con el fin de reforzar la actividad de composición escrita. Así mismo, el nivel de motivación por mejorar el aprendizaje del idioma es muy alto en general entre los estudiantes, pero su eficiencia es muy débil, especialmente cuando de escribir textos en inglés se trata. Por ende, para ensayar una estrategia didáctica nueva para ellos entonces se desarrollaron estas actividades estimulantes para mejorar esta habilidad comunicativa. En los talleres se emplearon diferentes tipos de estrategias directas e 
indirectas que permitieron estimular la memoria y son de orden cognitivo, compensatorio y de naturaleza afectiva y social que fundamentan una meta cognición que les permite a los estudiantes una retro alimentación para enriquecer sus escritos en la segunda lengua.

Dentro del marco de una investigación cualitativa, por medio de Análisis de Hatch comprende el uso de varias estrategias al estudiar un mismo fenómeno, por ejemplo, el uso de varios métodos (entrevistas individuales, grupos focales o talleres investigativos). Al hacer esto, se cree que las debilidades de cada estrategia en particular no se sobreponen con las de las otras y que en cambio sus fortalezas sí se suman. Se supone que, al utilizar una sola estrategia, los estudios son más vulnerables a sesgos y a fallas metodológicas inherentes a cada estrategia

\section{A continuación, se explica paso a paso, la tipología de Hatch.}

a) Identifica tipologías para ser analizadas. Se escogen las tipologías que van a ser utilizadas, para cuadrar el resto del análisis. Luego, el análisis solo tiene utilidad, cuando el agrupamiento inicial de datos y las estrategias iniciales son fáciles de identificar y justificar.

b) Lee los datos haciendo entradas relacionadas con su tipología. Después, que los conjuntos de tipologías han sido detectados, Hatch, recomienda que los datos sean leídos completamente. Es decir, la idea es encontrar y marcar esos lugares en los datos, en la cual evidencia relacionada, con la tipología particular sea encontrada y separar los datos más grandes de los pequeños basados en la tipología predeterminada. Entonces, el resultado será "racimos" grandes de datos en bruto, relacionados con las tipologías particulares diseñadas de todo el conjunto de datos originales.

c) Miran los modelos (patrones), relaciones, temas dentro de las tipologías. Debería ser creada para cada informante y como los datos exceptuados son leídos, se debería escribir una breve declaración de la idea principal de lo exceptuado, en la hoja de resumen. El objetivo es crear un resumen de lo que a menudo sería una gran cantidad de datos.

d) Lee las entradas por tipología. Codificando las entradas de acuerdo a los patrones identificados y manteniendo un expediente, llevando un registro de que entradas van y con cuáles elementos de sus modelos. Así pues, los patrones son irregulares, ellos vienen en varias formas, incluyendo semejanzas (cosas que suceden en la misma forma, diferencias (ellas suceden a menudo, rara vez,), secuencia (suceden en cierto orden), 
correspondencia (suceden en relación a otras actividades o eventos), causalidad (una aparece a causa de otra).

En cuanto a las relaciones, estas son enlaces. Spreadley (1979, p. 111) identificó muchas relaciones semánticas que pueden volverse la herramienta para identificar enlaces entre los elementos de los datos. Por otro lado, los temas son conceptos integradores, es decir, que ellos pueden ser definidos como declaraciones de significado que corren a través de todos o de la mayoría de los datos pertinentes (Ausubel, D. \& et al., 1976; Torres, T., 2003).

Estos datos buscan temas preguntados: ¿Qué declaraciones generales significativamente pueden ser hechas de todos estos datos juntos? La insatisfacción con el pago que se cree que se impregna, los parches de los profesores, quienes abandonan el campo, pero pueden tener datos que indique que otros temas también son importantes.

\section{e) Lee los datos, codificando las entradas de acuerdo a los patrones identificados $y$} manteniendo un expediente. Llevando un registro de que entradas van y con cuáles elementos de sus modelos. Ahora volvemos a los protocolos marcados y lea todos los dato marcados para sus patrones entre los orientadores y los estudiantes pueden suponer cuatro tipos de patrones de relación: psicólogo-cliente, consejero-estudiante, mentor monitoreado y padre-hijo. Entonces, él o ella leerá todos los datos resaltados que indican que las relaciones psicólogo-cliente se codificarán con algún tipo de marca que lo distingue de las otras categorías. Al mismo tiempo, recomiendo hacer un registro simultáneo de los elementos relacionados a esa categoría donde son encontrados los datos.

\section{f) Decida sí sus modelos son apoyados por los datos y la búsqueda de los datos para}

el no ejemplo de los patrones. Es evidente que no todos los extractos encajarán perfectamente en sus categorías, habiendo codificado todo lo que pueda, ahora es necesario hacer un juicio sobre sus categorías no están justificadas por los datos. Por un lado, tu juicio se basará en qué tan bien los datos codificados se ajustan a las categorías que usted ha intentado. Otro aspecto del problema es decidir si los datos no codificados contienen ideas que son diferentes o contradictorias con lo que ha propuesto.

g) Busque relaciones entre los patrones identificados: Los procedimientos anteriores serán realizados con todas las categorías iniciales identificadas en el primer paso. De esta manera, el resultado será un conjunto de patrones, relaciones y temas que tienen para este punto, analizado por separado. Así, la tarea ahora es regresarse al análisis del 
individuo que se han completado y buscar conexiones a través de lo que se ha encontrado. Desde luego, este proceso a menudo se puede ayudar haciendo algunos tipos de representación visual de las categorías exploradas hasta ahora, y luego ver qué relaciones pueden existir entre categorías o entre ellas.

Miles y Huberman (1994) describe tales representaciones visuales como "pantallas de datos”. Qué presentan información de manera sistemática, para que el usuario pueda sacar conclusiones válidas y tomar las medidas necesarias. Por ejemplo, en un estudio de individuos que dejan la enseñanza antes de edad de jubilación, haciendo cuadros para categorías como bajos salarios, bajo estatus, bajo sentido de eficacia y bajo sentido autonomía podrían conducir al descubrimiento de conexiones que dan un sentido mucho más rico de por qué los maestros se van de los elementos de informe por separado.

g) Escribe sus modelos como generalizaciones de una oración. Mientras los investigadores experimentaron, ellos pudieron ver esto como limitante es y/o innecesario, esta estrategia ha sido muy útil para mis alumnos que solo están aprendiendo a hacer análisis de datos cualitativos, pues, si construye oraciones, lo obliga a organizar su pensamiento en una forma que usted y otros pueden entender. Entonces, resulta ser invaluable a medida que comienza a escribir sus hallazgos, pero sirve para dar cierre a sus análisis. Esto indica que, una generalización expresa una relación entre dos o más conceptos. Así mismo, expresar hallazgos como generalizaciones proporciona una sintaxis o dispositivo para asegurar que lo que se ha encontrado se puede comunicar a otros. Por ende, si los hallazgos no pueden expresarse como generalizaciones, las posibilidades son datos de análisis incompleto.

\section{h) Seleccione extractos de datos que respalden sus generalizaciones: en} preparación para escribir descubrimientos, el último paso en el modelo tipológico es volver a los datos para seleccionar ejemplos poderosos que se pueden usar para hacer que su generalización cobre vida para sus lectores.

En los informes cualitativos, es habitual incluir datos, extractos que llevan a los lectores dentro de los contextos y les permiten escuchar las voces de los participantes. Por ello, es mejor seleccionar citas potenciales de los protocolos en este punto del proceso en lugar de volver a los datos cuando estás involucrado en su escritura y los datos son fríos. En otras palabras, este es un lugar donde puedes regresar al registro que hizo cuando codificaba los datos. Si se siguen las sugerencias anteriores se hizo una nota de dónde 
los extractos relacionados se ubicaron categorías, encontrar citas destacadas no será difícil.

\section{RESULTADOS Y DISCUSIÓN}

El uso de las estrategias pedagógicas en la escritura del inglés, es esencial al momento de aprender una segunda lengua. Es decir, que el manejo de estrategias específicas hace factible que el docente prepare mejor sus evaluaciones y obtenga emplear grandes resultados. Incluso, a los aprendices les permite a adoptar las mejores y más exitosas estrategias de composición, que por ello se reflejan en mejores producciones textuales. Igualmente, entre los hallazgos más importantes en las estrategias, está la reescritura que agrupa destrezas como la toma de notas en clase, redacción de textos, elaboración de frases cortas, ejercicios escritos en clase, a partir de tema dado por el docente en clase.

Así mismo, se encontró que un aspecto que podría ser muy útil para los aprendices, es el interactuar con hablantes nativos, pero desafortunadamente en la zona donde está ubicada la institución (Puerto Santander) no es posible tener esa oportunidad, por varios motivos (zona de difícil acceso, orden publico complicado, poca conectividad de internet) ajenos a los estudiantes y al docente, por lo cual se estimuló y recomendó utilizar los medios de comunicación disponibles para que buscaran y revisaran sitios de habla y escritura inglesa.

Otro aspecto concluyente, fue el uso del diccionario bilingüe. Es una estrategia que los estudiantes la consideran de fácil acceso y muy efectiva al momento de escribir en inglés. Por ello, este es una de las herramientas pedagógicas con la que la mayoría de los discentes cuentan en todo momento que practican la escritura. De otro lado, está la memorización, donde se encuentran estrategias como exposiciones en el tablero, elaboración de textos a partir de un grupo de palabras, talleres grupales e individuales, socialización de actividades entre los alumnos y el docente. Cabe resaltar que los resultados evidencian que cuando se usaban estas estrategias siempre fueron muy productivos y ellos siempre mostraron empatía hacia estas.

Otro punto relevante es la retroalimentación, luego, es una de las estrategias que más usa el estudiante, quizá porque es de las más completas, es decir abarca desde el inicio del aprendizaje, siguiendo con su propia corrección y la de sus compañeros. Igualmente, este proceso hace que el estudiante se sienta seguro con su aprendizaje, también un aspecto a resaltar es el inicio del estudiante a estudiar inglés en el bachillerato parece ser 
tardío. Esto indica que, la mayoría empiezan en sexto grado, aunque uno que otro lo hacen en algún grado de primaria, la cual es una gran desventaja el no comenzar este aprendizaje, en los primeros años de estudio.

En cuanto al uso de las TIC, es muy preocupante que los estudiantes no cuenten con un recurso tecnológico tan importante y tan productivo como un computador individual o uno en el hogar, ni un teléfono celular de última generación, o algún otro dispositivo que pudiera ser aprovechado por los estudiantes de la institución, donde se hizo el proyecto. A esta situación se suma que, en la zona de influencia del estudio la conectividad a internet es casi nula.

La habilidad escrita en los estudiantes se desarrolla en la medida que el docente le da la importancia que ésta amerita en el aula y la muestra de tal manera que logre atraer la atención y el deseo de aprender del estudiante. De esta manera, es necesario utilizar técnicas que admitan incluir en el campo de la escritura de pequeños textos de acuerdo a las habilidades de los futuros escritores y los estándares indicados para cada grado en el empleo de la segunda lengua.

De hecho, se detectan efectos en el aprendizaje y en la enseñanza de la escritura. Por consiguiente, una consideración del proceso de escritura y cómo hablar y escribir están: relacionados entre sí, esencialmente en un mundo de medios de comunicación variable. Estos indican que, no es solo de importancia académica; luego, tiene consecuencias para la forma en que enseñamos la escritura. Por ende, muchos modelos tradicionales, no agregaron los tipos de conocimiento en el proceso de escritura que hemos estado discutiendo.

También, por ejemplo, los estudiantes escriben una composición en el aula, con el docente corrige y devuelve el día siguiente relleno con tinta roja. Además, los estudiantes colocan los trabajos corregidos en sus carpetas y esporádicamente los revisan nuevamente;

Por consiguiente, el taller de escritura creativa puede ser aplicado en los diferentes niveles de formación, ya sea para impulsar la escritura o para superar esta habilidad en lengua materna o extranjera. En otras palabras, este debe tener en cuenta las necesidades comunicativas y agrado de los estudiantes, es por ello que el material que se escoja para las etapas, los talleres deben ser estimulantes y significativos, de esta manera la intervención será mayor y más gratificante. 
De igual forma, para lograr que nuestros estudiantes ejerciten habilidades de escritura es importante que investiguemos sobre nuestro rol como docentes.

a) Para el desarrollo de la producción escrita debe haber direcciomiento de parte del docente al interior del curso y enlazado con el tipo de producciones evaluadas.

b) Es importante el direccionamiento por parte del docente, lo cual produce un ambiente de confianza y seguridad en los estudiantes.

c) También un aspecto relevante es el comienzo del estudiante a estudiar inglés en el bachillerato. Desde luego, la mayoría inician en sexto grado, aunque uno que otro lo hace en algún grado de primaria, entonces, es una gran desventaja la acción de no dar comienzo a este aprendizaje, en los primeros años de estudio.

Cabe resaltar, una forma de incentivar la redacción, la consideración y la revisión es hacer que los estudiantes compartan en la escritura colaborativa. Por ejemplo, un grupo de estudiantes que elaboran juntos en un escrito pueden contestar a las ideas de otros compañeros, tanto en vocablos de lenguaje como de contexto), haciendo sugerencias, cambios y aportando así al éxito del producto finalizado.

Por ello, antes de hacer que los estudiantes escriban, podemos aninarlos a pensar en lo que van a escribir, planeando el contenido y la secuencia de lo que escribirán en papel o escribirán en la computadora. De nuevo, hay varias formas de hacerlo, incluso a escala, como, lluvia de ideas en la que los estudiantes en parejas o grupos muestren tantas ideas como puedan, a través de una discusión) para desempeñar tareas mis dirigidas. Es decir, donde el profesor o el libro del curso involucren una serie de ejercicios que dirigen a los estudiantes a planear una tarea futura. Igualmente, en el momento dado que los estudiantes planean, podemos animarlos a pensar no solo en expresar lo que quieren decir, sino también sobre el objetivo de su escritura y para quién es lo que están escribiendo.

De igual importancia, los estudiantes que se recreen con las lecciones de redacción de párrafos, deberán ser impulsados a reflexionar sobre aquello que ellos escribieron, en otras palabras, aprender a manejar los primeros borradores como primeros intentos y no como productos finales. Así mismo, es posible que deseemos reforzarlos en el uso y la respuesta a los símbolos de corrección. Por lo tanto, es posible que les ofrezcamos listas de comprobación para que empleen la verificación de lo que han escrito con el fin de realizar correcciones. 
De hecho, una forma de motivar la redacción, la consideración y la revisión es hacer que los estudiantes compartan en la escritura colaborativa. Por ejemplo, un grupo de estudiantes que trabajan juntos en un escrito pueden contestar a las ideas de los demás, tanto en términos de lenguaje como de contexto), haciendo sugerencias, cambios y aportando así al éxito del producto final.

Igualmente, para que un modelo de escritura de proceso se desempeñe bien, algunos docentes pueden necesitar reflexionar la manera en que pueden actuar ante el trabajo de sus alumnos. Lo cual indica que, en lugar de hacer enlaces a una narración terminada, a veces necesitarán responder a un trabajo en avance. Incluso, esto puede inferir hablar con estudiantes individuales acerca de un primer, segundo, tercer y cuarto borrador, mientras que otros individuos del grupo están trabajando en su propio escrito.

Fluctuosamente, los docentes pueden leer un borrador y luego crear sugerencias por escrito sobre cómo se podría reorganizar el texto. Por ende, el proceso de escritura es una forma de observar aquello que la gente realiza cuando ellos elaboran un texto escrito; pues, se ha visto que es recursivo y puede involucrar muchos cambios de dirección mientras el escritor corta y varía entre los cuatro fundamentos principales del proceso, aunque, la escritura de procesos puede no ser la respuesta en todas los momentos de aprendizaje

\section{CONCLUSIÓN O CONSIDERACIONES FINALES}

Para lograr que nuestros estudiantes ejerciten habilidades de escritura es importante que los docentes nos evaluemos constantemente.

Para el desarrollo de la Producción escrita debe haber acompañamiento de parte del docente al interior del curso y enlazar con el tipo de producciones evaluadas.

Es importante el apoyo por parte del docente, lo cual genera un ambiente de confianza y seguridad en los estudiantes.

También un aspecto a resaltar es el inicio del estudiante a estudiar inglés en el bachillerato. La mayoría empiezan en sexto grado, aunque uno que otro lo hacen en algún grado de primaria, luego, es una gran desventaja el no comenzar este aprendizaje, en los primeros años de estudio

El taller de escritura creativa es un espacio favorable para el inicio y mejora de la producción escrita, así mismo, su ambiente propicia la creación al dar mayor importancia al fondo y no a la forma del texto. Esto contribuye a que el estudiante dé 
rienda suelta a su imaginación relegando a un segundo lugar bloqueos mentales que dificultan la escritura.

Igualmente en él se fortalece el trabajo cooperativo ya que los estudiantes interactúan entre sí de manera respetuosa, con el fin de mejorar sus escritos ya sea en el proceso de creación o en el de corrección.

El uso de las estrategias pedagógicas en la escritura en inglés, es primordial al momento de aprender una segunda lengua. Por ello, el uso de ellas, hace posible que el docente planee mejor sus actividades y así mismo, orienten a los aprendices a adoptar las mejores y más exitosas estrategias, que a la postre se reflejan en mejores producciones textuales.

\section{REFERENCIAS}

Ausubel, D., Novak, J., \& Hanesian, H. (1976)... Psicología educativa: un punto de vista cognoscitivo, $1(2)$ 53-106.). https://www.arnaldomartinez.net/docencia_universitaria/ausubel02.pdf

Aparicio, M. \& Navarro, E. (2017). Accesibilidad universal: sentido normativo e implicaciones en la educación y la práctica profesional. https://www.cedd.net/redis/index.php/redis/article/view/361

Cassany, D. (1990). Enfoques didácticos para la enseñanza de la expresión escrita. Comunicación, lenguaje y educación, 2(6), 63-80. http://hdl.handle.net/10230/21225

Crystal, D. (2003). English as a global language. Ernst Klett Sprachen. http://culturaldiplomacy.org/academy/pdf/research/books/nation_branding/English_ As_A_Global_Language_-_David_Crystal.pdf

Denzin, N. \& Lincoln, Y. (2005). Introducción: La disciplina y práctica de la investigación cualitativa. http://metodo3.sociales.uba.ar/wpcontent/uploads/sites/169/2014/10/Denzin-

Galán, M. (2009). El cuestionario aplicado en la investigación. http://manuelgalan.blogspot.com/2009/04/el-cuestionario-en-la-investigacion.html

Galeano, M. (2018). Estrategias de investigación social cualitativa: el giro en la mirada. Fondo Editorial FCSH. https://libreriasiglo.com/categoria/47258estrategias-de-investigacion-social-cualitativa-el-giro-en-lamirada.html\#.YC5_wehKjI 
Guano, D., Peñafiel, M., Yerovi, E., \& Andrade, L. (2017) El Aprendizaje significativo Como estrategia de estimulación de la escritura del idioma inglés en educación general básica.

Hatch, J. (2002). Doing qualitative research in education settings. Suny Pres

Hernández, R., Fernández, C., \& Baptista, P. (2006). Análisis de los datos cuantitativos. Metodología de la investigación, 407-499.

MEN (2006) Decreto 4675 de diciembre 28 de 2006 (En linea) Por el cual se modifica la estructura del Ministerio de Educación Nacional, y se dictan otra disposiciones.

Disponible en: https://www.mineducacion.gov.co/1759/w3-article119464 html?

Nichol (2006) La accesibilidad en evolución, la adaptación persona-entorno y su aplicación al medio residencial en España y Europa

Miles, M. \& Huberman, A. (1994). Qualitative data analysis: An expanded sourcebook. Sage.

Rincón, A. (2013). Fortalecimiento de la escritura en inglés en estudiantes de educación media de una institución pública. Villavicencio, Colombia. https://repositorio.tec.mx/handle/11285/626565

Sabino, C. (1992). El proceso de investigación, Ed. Panapo, Caracas. https://metodoinvestigacion.files.wordpress.com/2008/02/el-proceso-deinvestigacion_carlos-sabino.pdf

Sampieri H. (2006) Método cualitativo de investigación. Quinta Edición. Limusa: México.

bposgrado.org/material_seminarios/HSAMPIERI/Metodologia\%20Sampieri\%205a \%20edicion.pdf

Spradley, J. (1979).The ethnographic interview, 1, 44-61.

Torres, T. (2003). El aprendizaje verbal significativo de Ausubel. Algunas consideraciones desde el enfoque histórico cultural. Universidades, (26), 37-43.

Vargas, I. (2012). La entrevista en la investigación cualitativa: nuevas tendencias y retos... Revista electrónica calidad en la Educación Superior, 3(1), 119-139. https://revistas.uned.ac.cr/index.php/revistacalidad

Veloz, S. (2015). El trabajo autónomo en los estudiantes de inglés como parte de sus procesos de aprendizaje en la habilidad escrita, en el semestre" A" año 2014 de los estudiantes en el Centro de Idiomas Extranjeros de la Universidad Católica de 
Desarrollo de la

Santiago

de

Guayaquil.

http://www.lareferencia.info/vufind/Record/EC_cb99d978c18afae5236b3e51a05faa 27

Villegas, J., García, A., \& Escalera, M. (2016). Variables que influyen sobre el aprendizaje del inglés como segunda lengua. Revista Internacional de Lenguas Extranjeras/International Journal of Foreign Languages, (5), 79-94. https://core.ac.uk/download/pdf/83007833.pdf 